\title{
PATTERN, INDICATIONS AND REVIEW OF COMPLICATIONS OF NEONATAL BLOOD TRANSFUSION IN IBADAN, SOUTHWEST NIGERIA
}

\author{
A.I. Ayede.* and T.S. Akingbola.** \\ *Department of Paediatrics, University College Hospital, Ibadan, Nigeria. \\ **Department of Haematology, University College Hospital, Ibadan, Nigeria
}

Correspondence:

Dr. Adejumoke I. Ayede

Department of Paediatrics

University College Hospital

Ibadan,

Nigeria.

e-mail: idayede@yahoo.co.uk

$+2348033740698$

\begin{abstract}
Background and objectives: There is a huge need for blood transfusion in the newborn particularly due to the reduced marrow activity in the neonatal period. Despite widely use of blood products in the neonatal period, there is paucity of local data on the pattern, indications and reactions to blood transfusions in Nigerian newborns. This study evaluates the blood transfusion indications and patterns in special care baby unit and $\mathrm{C}^{2 \text { nd }}$ of University College Hospital, Ibadan, Nigeria. Methodology: A cross sectional study was carried on the recruited newborns and structured questionnaires were used to obtain bio data, medical problems, indications for blood transfusion, type of blood products transfused and clinical signs. Urinalysis was performed out before and after the transfusion.

Results: A total of 100 neonates were recruited into the study with a male: female ratio of $(M: F=1: 1)$. The age range was $2-34$ days and their weight ranged between $0.8 \mathrm{~kg}$ to $3.6 \mathrm{~kg}$ with a mean weight of 1.64 $\mathrm{kg}$. The main indications for transfusion were anaemia from prematurity \& neonatal sepsis(NNS) 46\%; (red cell replacement), NNS, Disseminated intravascular coagulation(DIC) \& anaemia 24\%; (partial exchange + top up + Fresh frozen plasma), neonatal jaundice(NNJ) \& anaemia 14\%; (whole blood), NNJ, NNS + anaemia 6\%(Blood transfusion + Fresh frozen plasma), NNS + anaemia 10\% (whole blood). Conclusion: Blood transfusion is still frequent in the study area and prematurity, neonatal sepsis and jaundice rank high in the indications. Transfusion reactions are rare in the evaluated neonates.
\end{abstract}

\section{INTRODUCTION}

Blood transfusion is the process of infusion of blood and blood products into an individual's circulatory system. Blood transfusion is used in a variety of medical conditions to replace lost components of the blood. Component therapy whereby components of the blood such as red cell concentrate, fresh frozen plasma, cryoprecipitate, and platelet concentrate are infused as indicated, has overtaken the old practice of whole blood infusion. The science of blood transfusion dates back to the first decade of the 19th century, with the discovery of distinct blood types leading to the practice of mixing some blood from the donor and the recipient before the transfusion (ref). Blood transfusion need in the neonate is huge because of reduced marrow activity in the neonatal period. ${ }^{1}$ Other indications include replacement of losses due to blood loss in the course of taking blood samples for various tests, correction of anaemia, exchange blood transfusion or partial exchange transfusions for removal of bilirubin, removal of antibodies and replacement of red cells. Blood products such as platelets concentrates and fresh frozen plasma can also be transfused in septicaemic neonates with DIC and neonates with bleeding diathesis ${ }^{2}$.In spite of wide use of blood and blood products in the neonatal period, there is paucity of local data on the pattern, indications and reactions to blood transfusions in Nigerian newborns. This study evaluates the blood transfusion indications and patterns in neonates admitted to neonatal wards of University College Hospital, Ibadan, Nigeria.

\section{MATERIALS AND METHODS}

\section{Study site}

The study was carried out at the Special Care Baby Unit (SCBU) and C1 ${ }^{2 n d}$ wards of the Department of Paediatrics, University College Hospital (UCH) Ibadan, Nigeria. Ibadan is the capital of Oyo state located in the southwest zone of Nigeria. The UCH serves as the referral centre to all primary and secondary health facilities located in Oyo state and even other states in the southwest and other zones. The SCBU admits 
babies within the first 48 hours of live that are delivered within $\mathrm{UCH}$ or referred from outside while $\mathrm{C}^{2 \text { nd }}$ admits both newborns and children up to 1 year of age.

\section{Study Subjects}

All the patients involved in this study were neonates admitted either into the SCBU or $\mathrm{C}^{2 \text { nd }}$ of $\mathrm{UCH}$ and received blood or blood products transfusion for medically indicated reasons. All the blood and blood products were screened and processed by the Blood Bank of UCH, Ibadan.

\section{Study Type and Methods}

The study was a cross sectional study carried out on the recruited newborns in SCBU and $\mathrm{C}^{2 \text { nd }}$ of $\mathrm{UCH}$, between March and October 2010. Structured questionnaires were used to obtain bio data, medical problems, indications for blood transfusion, type of blood products transfused and the frequency, clinical signs before, during and after blood transfusion. Informed consent was obtained from the care givers of the recruited patients. The clinical signs were evaluated by Paediatrics Resident Doctors and a Consultant Paediatrician (AIA) in charge of SCBU. All blood products were prewarmed before transfusion and $1 \mathrm{ml}$ of calcium gluconate was given per every $100 \mathrm{mLs}$ of blood exchanged. Urinalysis was performed on all recruited neonates using multistix urine strip before and 12-24 hours post transfusion. Dual data entry was carried out by using Epi Data version 8 to enable data check, cleaning and verification. All the data were subsequently transferred into Statistical Package for Social Sciences (SPSS) version 17 for analysis. Frequencies were generated for categorical variables and compared using pie and bar charts.

\section{RESULTS}

\section{Patients Distribution}

A total of 324 neonates were seen during the period of the study out of which 100 neonates $(30.8 \%)$ had one blood product or the other and hence, were recruited into the study. The male: female distribution was 50: $50(\mathrm{M}: \mathrm{F}=1: 1)$ while their ages were between 2-34 days and their weight ranged between $0.8 \mathrm{~kg}$ to $3.6 \mathrm{~kg}$ with a mean weight of $1.64 \mathrm{~kg}$. The distribution of newborns according to age groups $0-7,8-14,15$ $21,22-28, \geq 29$ days was $19.4 \%, 31.6 \%, 24.5 \%, 17.3$

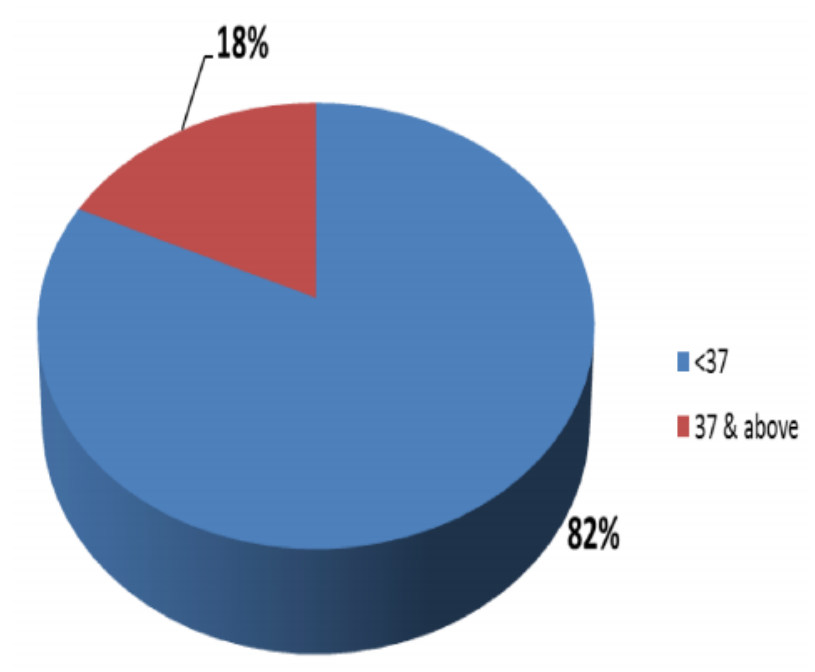

Figure 1: GA distribution

$\%$ and $7.1 \%$ respectively. Majority of the patients were preterms $(82 \%)$. The gestational age and postnatal age distribution is as shown in figures 1 and 2 .

\section{Type of blood transfusion}

Forty six percent $(46 \%)$ had red cell replacement, $24 \%$ had partial exchange + top up + fresh frozen plasma, $30 \%$ had whole blood + Fresh frozen plasma. Ninety percent $(90 \%)$ of the patients who had exchange blood transfusion (EBT) were preterm. Platelet rich plasma or platelet concentrate were not available for all indicated cases during the period of the study.

\section{Blood group of babies and Donor}

As shown in figure 3 , the pattern of blood groups of the babies were: $\mathrm{O}=43.9 \%, \mathrm{~B}=13 \%, \mathrm{~A}=40 \%$ \& $\mathrm{AB}=2 \%$. The frequency of the blood groups of the donor units were $\mathrm{O}=65 \%, \mathrm{~B}=12 \% \mathrm{~A}=23 \%$.

\section{Indications for transfusion}

The patients were categorized based on the clinical condition and the type of blood products received. The main indications for transfusion as shown in figure 4 were anaemia from prematurity, anaemia \& Neonatal sepsis(NNS) 46\%; (red cell replacement), NNS, Disseminated intravascular coagulopathy (DIC) \& anaemia 24\%; (partial exchange + top up + Fresh frozen plasma), Neonatal Jaundice(NNJ) \& anaemia 


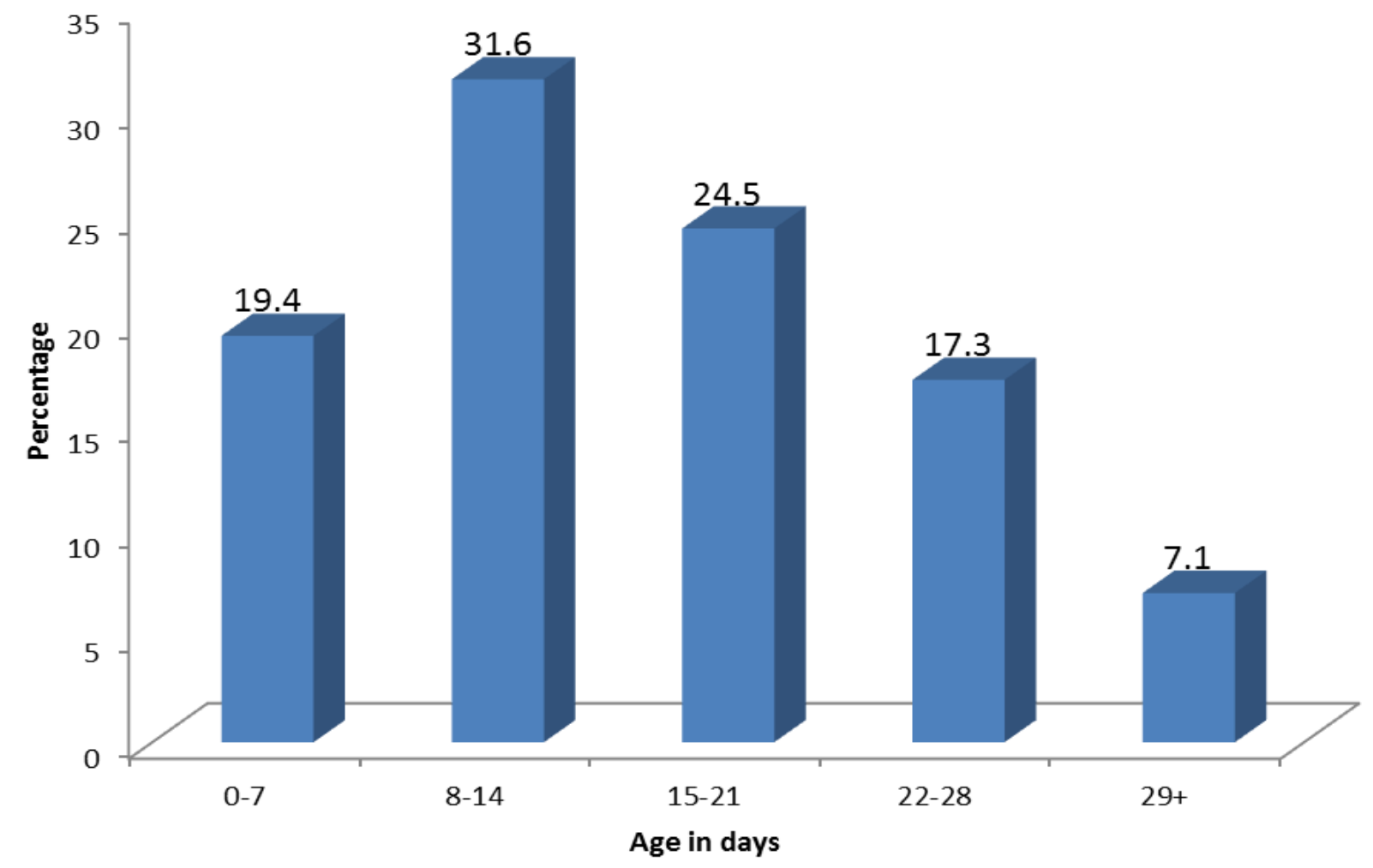

Figure 2: Age distribution of the neonates

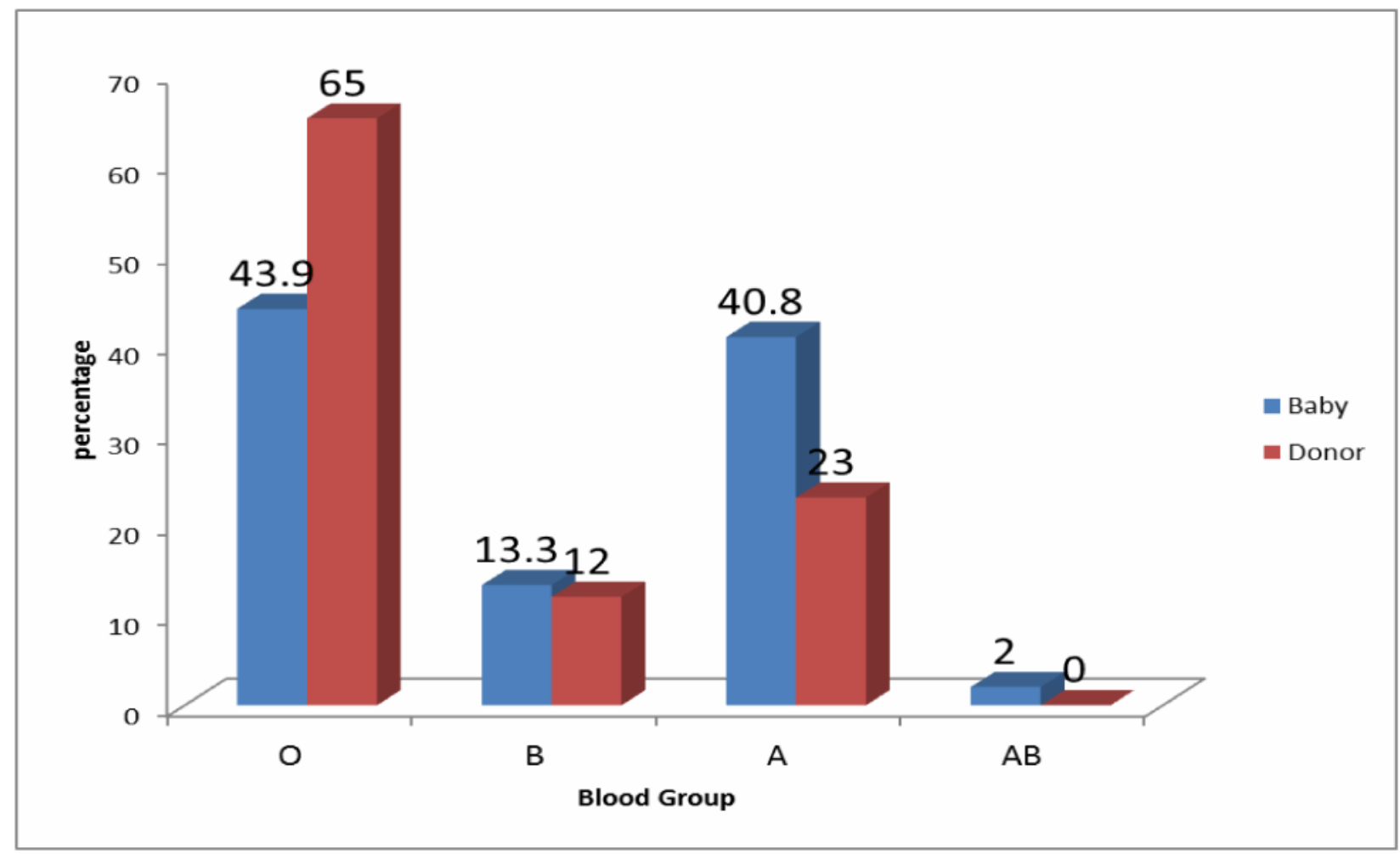

Figure 3: Blood group of baby versus blood group of donor 


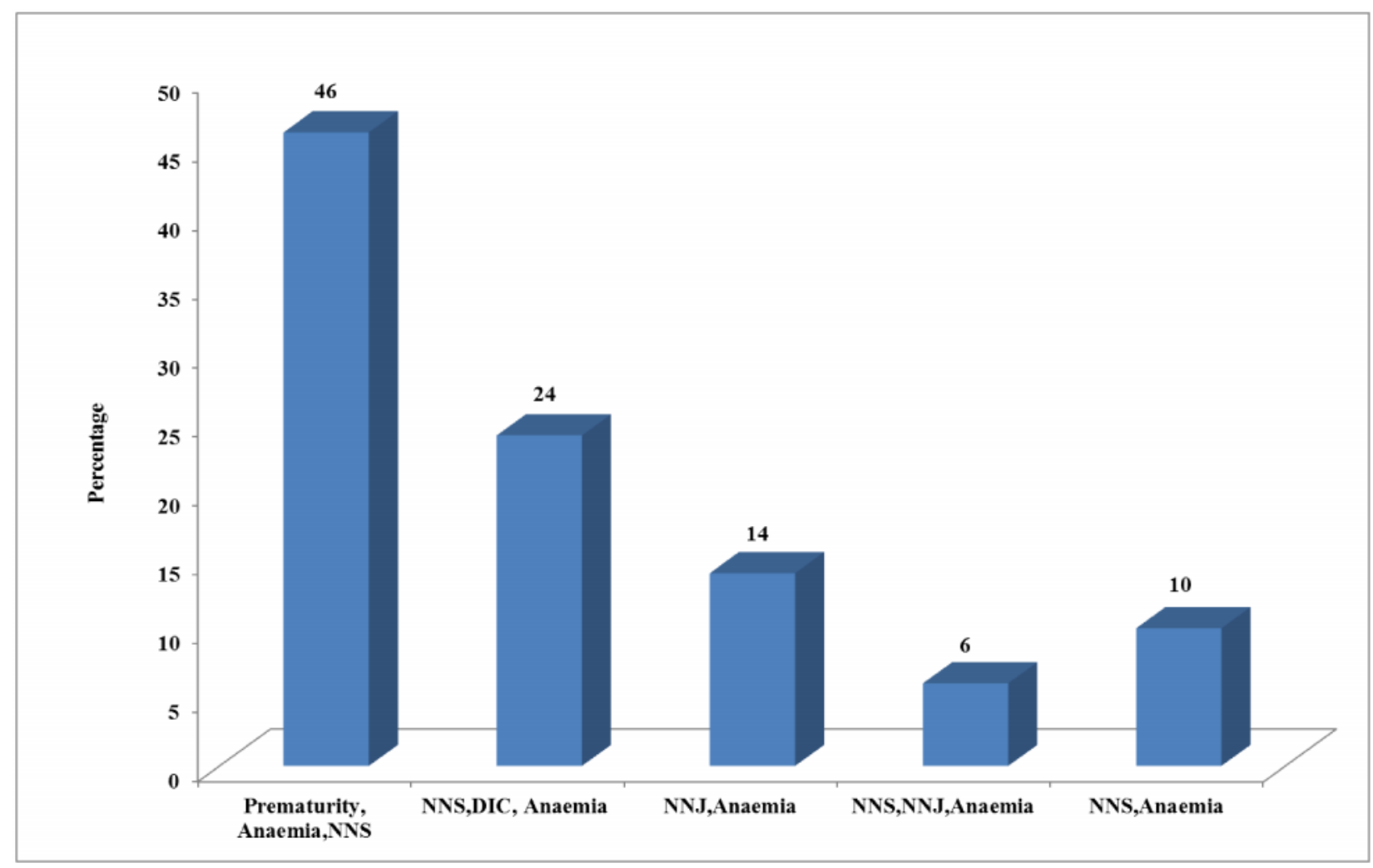

Figure 4: Indications for blood transfusion

14\%; (whole blood), NNJ, NNS + anaemia 6\% (Blood transfusion + Fresh frozen plasma), NNS + anaemia $10 \%$.

\section{Blood transfusion reactions}

There were no noticeable rigor, rash, jaundice (post transfusion), oliguria or haematocrit drop following transfusion but $5 \%$ had fever (all temperatures were not more than $2^{\circ} \mathrm{C}$ above preceding levels) during transfusion. Urine analysis showed evidence of haemosiderin and haematuria in 4 cases that also had DIC.

\section{DISCUSSION}

Transfusion practices for high risk infants differ among neonatal intensive care units. ${ }^{2-9}$ These practices vary as a function of birth weight, gestational age and severity of illness. ${ }^{3-4}$ The safest blood transfusion is the one not administered. When a transfusion is needed, it is important for both physicians and nursing staff to be aware of its potential acute and delayed adverse effects. Adverse consequences may be minimized through early recognition and prompt therapeutic intervention. Hence, all the babies in this study had full clinical evaluation done pre, intra and post blood transfusion. In addition to the issues concerning blood transfusions in general, special considerations apply to transfusing neonates. Neonates do not produce red blood cell (RBC) antibodies; any antibodies present are of

maternal origin. ${ }^{10}$ Young infants' plasma may contain maternal antibodies to $\mathrm{ABO}$ blood group antigens instead of the naturally occurring anti-ABO antibodies normally present in older patients. This complicates blood bank testing and selection of appropriate blood components for transfusion. Conversely, blood bank testing is simplified by the fact that neonates do not make antibodies to minor erythrocyte antigens. ${ }^{10}$ All blood transfusions carried out in these patients had the grouping and cross matching of both mother and baby samples. Cellular blood components may be irradiated to prevent transfusion-associated graftversus-host disease, a syndrome that can affect immunocompromised patients such as premature infants. However, irradiation accelerates the leakage of potassium out of stored red blood cells (RBCs), increasing the risk or transfusion-induced arrhythmias from hyperkalaemia and hypocalcaemia. Irradiation was not done for any of the blood transfused in these patients because this was not available.

Premature infants are among the most frequently transfused groups of patients, usually receiving red cells. ${ }^{3.4}$ This was the case here where $82 \%$ of the patients transfused were preterms. The vast majority of preterm infants experience an exaggerated physiologic anaemia primarily occurring in infants born at fewer than 32 weeks gestation, the haemoglobin can fall to as low as $7 \mathrm{~g} / \mathrm{dL}$ by $4-6$ weeks of age. This normocytic anaemia is not associated with deficiencies of iron, protein, vitamin E, vitamin B12, or folate and usually improves spontaneously with time, but many 
symptomatic infants may require treatment. The preterm babies have a higher chance of being admitted with serious illnesses that will necessitate blood investigations, therefore are predisposed to iatrogenic anaemia. They are also more likely to be ill. Particularly, they may have neonatal septicaemia which may also make them prone to develop bleeding diathesis. Another major reason is their poor bone marrow response to anaemia. In sick preterm infants, anaemia of prematurity can be accentuated by nonphysiological anaemia, characterized by a decreasing haematocrit, reticulocytopaenia, bone marrow hypoplasia, and endogenous erythropoietin concentrations that are inappropriately low relative to the degree of anaemia. ${ }^{3,4}$ The most common cause of nonphysiological anaemia is blood loss, which may be acute or chronic. A common and potentially avoidable cause of postnatal blood loss is iatrogenic as a result of frequent blood sampling (phlebotomy) in sick preterm infants. ${ }^{3-4}$

About $50 \%$ of the babies transfused were within the first 2 weeks of life and this is in keeping with the fact that many babies that are ill will be ill in first few days of life. Also, the blood group of the donors and recipient are in keeping with the pattern general population. This study also showed that the various indications for transfusion are a reflection of the national neonatal burden which includes prematurity, neonatal sepsis and jaundice ${ }^{11}$. Late presentation to referral centres with resultant worsening of clinical condition and non-availability of early intervention methods may be responsible for the high use of blood products in these patients. Lack of prompt management due to high cost of treatment and out of pocket payment also might have played significant role in the clinical states of the babies evaluated in this study ${ }^{12}$. This may be responsible for rapid deterioration of the clinical state leading to worsening of jaundice and development of disseminated intravascular coagulopathy. The multiple clinical conditions that these patients presented with are also reflections of the severity of their clinical states.

The common blood transfusion related reactions are: Febrile, non-haemolytic transfusion reaction (FNHTR), allergic/urticarial/anaphylactic reaction, acute non immune transfusion reactions and fluid overload. Acute haemolytic transfusion reactions are the second commonest cause of transfusion-related fatality in adult patients, but these are rare in neonates. ${ }^{13}$ The symptoms of haemolysis typically seen in older patients, such as fever, hypotension, and flank pain, usually are not identified in the neonatal patient. In the neonate, an acute haemolytic event may be characterized by increased plasma free haemoglobin, haemoglobinuria, increased potassium concentration, and decreased $\mathrm{pH}$.
Results of the direct antiglobulin (Coombs) test may confirm the presence of an antibody on the RBC surface. Treatment is supportive to maintain blood pressure and renal perfusion with intravenous normal saline at 10 to $20 \mathrm{~mL} / \mathrm{kg}$ and diuresis with furosemide. Prevention is aimed at minimizing human errors and improving patient safety by strict regulations on patient identification before blood is drawn or administered. None of the patients seen in this study developed acute haemolytic transfusion reactions or urticarial rash.

Febrile nonhaemolytic transfusion reactions (FNHTR) are suspected in the absence of haemolysis with an increase in body temperature of less than $2^{\circ} \mathrm{C}$. FNHTR occur in $0.1 \%$ to $1 \%$ of transfusions in adults. The temperature rise is mediated by inflammatory cytokines (interleukin [IL]-1, IL-6, IL-8, tumor necrosis factor) released from white blood cells (WBCs) during storage of the blood component or caused by preformed recipient antibodies reacting with WBCs in theinfused component. Prestorage leukoreduction of RBCs and platelets reduces the incidence of FNHTR. ${ }^{13}$ Volume reduction of platelets also may reduce the incidence of these reactions. For reactions associated with a temperature rise of greater than $2^{\circ} \mathrm{C}$ or with hypotension, bacterial contamination also should be suspected and a Gram stain and microbial culture performed on the remaining blood product. Five per cent of the babies in this study had increase in temperatures of less than $2^{\circ} \mathrm{C}$ during the blood transfusion. The rate is higher than the usual rates in newborn. ${ }^{13}$ The high incidence of neonatal sepsis present in this cohort of babies studied might be responsible for the temperature rise. However, a small percentage of these babies may still be due to FNHTR. Allergic reactions are rare in neonates. They occur when a patient has preformed immunoglobulin (Ig) E antibody against an allergen in the donor plasma. Residual cytokines or chemokines (eg, RANTES) released by stored platelets also may contribute to allergic reactions. Most reactions respond to antihistamines. Severe anaphylactic reactions are rare; some are related to anti-IgA antibodies. These severe reactions are treated with epinephrine, steroids, or both as well as intubation and vasopressors if needed. Patients who have a history of anti-IgA antibodies or anaphylaxis to blood transfusion should receive washed cellular products. ${ }^{14}$ None of the patients had allergic reactions.

Transfusion-related acute lung injury (TRALI) is the most common cause of transfusion-related fatality but often remains unrecognized. The recommended diagnostic criteria for TRALI are the acute onset of hypoxemia with bilateral infiltrates on chest radiograph within 6 hours of a blood transfusion and no evidence 
of circulatory overload. Patients who have circulatory overload respond to diuresis, but those who have TRALI do not. The treatment of TRALI is oxygen support and mechanical ventilation, resulting in recovery within 96 hours for most patients. None of the patients had evidence of circulatory overload throughout the transfusion period.

It is hypothesized that TRALI may be the result of two cumulative events: the first linked to the patient, such as underlying sepsis, trauma, hematologic disease, or postsurgical status, and the second being haemolysis related to T-antigen activation is a rare complication of sepsis and necrotizing enterocolitis (NEC) in infants. The enzyme sialidases released by bacteria can alter RBC membranes by cleaving sialic acid residues, thereby exposing ("activating") the T antigen. Most plasmacontaining components contain naturallyoccurring anti$T$ agglutinins that can cause haemolysis of T-activated cells. Although T activation has been detected on the RBCs of up to $30 \%$ of neonates who have NEC, clinical haemolysis rarely is observed. ${ }^{15,16}$ Four percent of the patients had haemosiderin and haematuria but without clinical evidence of worsening haemolysis.

Neonates are at increased risk of fluid overload from transfusion because the volume of the blood component issued by the Transfusion Service Unit may exceed the volume that may be transfused safely into neonates. Care should be taken to ensure that, in the absence of blood loss, volumes infused do not exceed 10 to $15 \mathrm{~mL} / \mathrm{kg}$. Metabolic complications are encountered primarily with massive transfusions $(>15$ to $20 \mathrm{~mL} / \mathrm{kg}$ ) or exchange transfusions. The Patients that had blood volumes of up to $15-20 \mathrm{~mL} / \mathrm{kg}$ all had intravenous laxis before the transfusion and none presented with fluid overload. Hypocalcaemia can result from large infusions of citrate, which prevents clotting in blood components by binding calcium. The most feared symptom of hypocalcaemia is myocardial depression. A prolonged QT interval may be observed on electrocardiography. Cardiac monitoring or regular checks of ionized calcium level are recommended in neonatal patients receiving massive transfusions. The patients that received EBT had $1 \mathrm{ml}$ of calcium gluconate infused after every $100 \mathrm{mls}$ of whole blood exchanged. Hyperkalaemia can occur with rapid or massive infusion of stored $\mathrm{RBCs}^{17}$. Washing to reduce supernatant potassium may be appropriate in massive transfusions in neonates. The quantity of free potassium is not clinically important for small-volume transfusions administered slowly (eg, 3 to $5 \mathrm{~mL} / \mathrm{kg}$ per hour $)^{17}$. Hypoglycemia and hyperglycemia both have been reported in association with neonatal transfusions. An inadequate infusion rate of glucose may result if other sources of glucose are discontinued during transfusion. Hypoglycemic episodes occur more commonly with transfusion of CPDA-1 RBCs rather than additive RBCs, which contain larger quantities of glucose. Large-volume transfusions of additive RBCs may cause transient hyperglycemia followed by rebound hypoglycemia from the insulin induced by the glucose load. ${ }^{18}$

The infusion of cold blood components in surgery or massive transfusion may cause hypothermia, which may be associated with hypoglycemia, apnea, and arrhythmia complicated by cardiac arrest. This can be prevented by using a monitored blood warming system with alarms. ${ }^{19}$ All the blood products given in these babies were pre-warmed so none of the complications were seen in them.

Transmission of infections such as HIV, Hepatitis B \& C, Cytomegalovirus (CMV), Syphilis etc are possible through blood transfusion. ${ }^{20}$ In the study centre, all blood/blood products are routinely screened for some of these infections. CMV screening is not routinely done in our Centre.

\section{CONCLUSION AND RECOMMENDATIONS}

Blood transfusions are common among hospitalized neonates, particularly in those of preterm birth. Clinicians should use a kinetic approach to diagnosing neonatal anaemia, taking full advantage of the data available on the routine $\mathrm{CBC}$ laboratory results. Every NICU should have erythrocyte transfusion guidelines, and these should be followed in order to avoid unnecessary and unhelpful erythrocyte transfusions. Despite significant improvement brought to blood testing and handling, there remain both infectious and non-infectious risks associated with allogeneic blood transfusions. Data analysis has demonstrated that the incidence of adverse events is greater in children and especially infants. The long-term repercussions may be significant in the paediatric population. Bloodconservation modalities can be used safely in paediatric patients. Combined techniques seem to be more effective than any single modality. No blood-sparing protocol would be complete and efficient without the use of an evidence-based transfusion algorithm. Studies have demonstrated that paediatric patients tolerate lower haemoglobin levels without incurring adverse events. Finally, intense research into manufacture of red blood cells by pluripotent stem cell is ongoing and promising. The development of alternatives to transfusions was initiated over 50 years ago and has still not come to fruition. Only a few products are currently undergoing phase III trials. ${ }^{21}$ 


\section{REFERENCES}

1. Monagle P, Andrew M. Developmental haemostasis; Relevance to newborns and infants. In: Nathan DG, Orkin SH, Ginsburg D, eds. Haematology of infancy and childhood. Philadelphia: WB Saunders, 2003: 121 - 168.

2. Ringer SA, Richardson DK, Sacher RA et al. Variations in transfusion practice in neonatal intensive care. Paediatrics 1998; 101: 194 -202.

3. Maier RF, Sonntag JS, Walka MM. Changing practices of red blood cell transfusions in infants with birth weights less than 1000 g. J Pediatr 2000;136:220-4.

4. Widness JA, Seward VJ, Kromer IJ et al. Changing patterns of red blood cell transfusion in very low birth weight infants. J Pediatr 1996;129:680-7.

5. Donovan EF. Practice variation: Implications for neonatal red blood cell transfusions. J Pediatr 1998;133:589-90.

6. Ringer SA, Richardson DK, Sacher RA, et al Variations in transfusion practices in neonatal intensive care. Pediatrics 1998;101:194-200.

7. Levy GJ, Strauss RG, Hume H, et al. National survey of neonatal transfusion practices: I. Red blood cell therapy. Pediatrics 1993;91:523-9.

8. Strauss RG. Red blood cell transfusion practices in the neonate. Clin Perinatol 1995; 22:641-55.

9. Bednarek FJ, Weisberger S, Richardson DK, et al. Variations in blood transfusions among newborn intensive care units. J Paediatr 1998;133: 601-7.

10. Floss AM, Strauss RG, Goeken N, Knox L. Multiple transfusions fail to provoke antibodies against blood cell antigens in human infants. Transfusion. 1986; $26: 419-422$

11. Saving newborn lives in Nigeria: Newborn health in the context of the integrated maternal, newborn and child health strategy, revised $2^{\text {nd }}$ edition 2011, 1- 120
12. Health Reform Foundation of Nigeria: Nigeria Health Review 2006; 1 - 343

13. Gorlin JB. Noninfectious complications of pediatric transfusion. In: Hillyer C, Strauss R, Luban N, eds. Handbook of Pediatric Transfusion Medicine. San Diego, Calif: Elsevier; 2004:317 -327

14. Wakamoto S, Fujihara M, Kuzuma K, et al. Biologic activity of RANTES in apheresis PLT concentrates and its involvement in nonhemolytic transfusion reactions. Transfusion. 2003;43:1038 1046

15. Boralessa $\mathbf{H}$, Modi $\mathrm{N}$, Cockburn $\mathrm{H}$, et al. RBC $T$ activation and hemolysis in a neonatal intensive care population: implications for transfusion 2002;42:1428 -1434

16. Engelfriet CP, Reesink HW, Blood transfusion in premature or young infants with polyagglutination and activation of the $\mathrm{T}$ antigen. Vox Sang. 1999;76:128-132

17. Galel SA. Therapeutic techniques: selection of blood components for neonatal transfusion. NeoReviews. 2005;6 :e351 -e355. Available at: http:/ Lneoreviews.aappublications.org/cgi/content/ full $/ 6 / 7 / \mathrm{e} 351$

18. Pisciotto PT, Luban NLC. Complications of neonatal transfusion. In: Popovsky MA, ed. Transfusion Reactions. 2nd ed. Bethesda, Md: AABB Press;2001:359 -394

19. Barcelona SL, Cote CJ. Pediatric resuscitation in the operating room. Anesthesiol Clin North Am. 2001;19:339-365

20. Dellinger EP, Anaya DA. Infectious and immunologic consequences of blood transfusion. Crit Care. 2004;8(suppl 2) :S18 -S23

21. Lavoie, J. Blood transfusion risks and alternative strategies in pediatric patients. Pediatric Anesthesia. 2011; 21: 14-24. 